\begin{tabular}{|c|l|}
\hline Title & Effect of indium doping on the transient optical properties of GaN films \\
\hline Author(s) & Kumano, H.; Hoshi, Ken-ichi; Tanaka, Satoru; Suemune, Ikuo \\
\hline Citation & $\begin{array}{l}\text { A pplied Physics Letters, 75(19), 2879-2881 } \\
\text { https://doi.org/10.1063/125178 }\end{array}$ \\
\hline Issue Date & 1999-11 \\
\hline Doc URL & http://hdl.handle.net/2115/5539 \\
\hline Rights & Copyright $\odot$ 1999 A merican Institute of Physics \\
\hline Type & article \\
\hline File Information & APL 75-19.pdf \\
\hline
\end{tabular}

Instructions for use 


\title{
Effect of indium doping on the transient optical properties of GaN films
}

\author{
Hidekazu Kumano, ${ }^{\text {a) }}$ Ken-ichi Hoshi, Satoru Tanaka, and Ikuo Suemune \\ Research Institute for Electronic Science, Hokkaido University, Sapporo 060-0812, Japan
}

Xu-Qiang Shen, Philippe Riblet, Peter Ramvall, and Yoshinobu Aoyagi

The Institute of Physical and Chemical Research (RIKEN), Wako, 351-0198, Japan

(Received 2 July 1999; accepted for publication 13 September 1999)

\begin{abstract}
We have investigated the effects of In doping on the optical properties of GaN films grown by gas-source molecular-beam epitaxy. Time-resolved photoluminescence was carried out to study the transient optical properties of the epitaxial films. In comparison to the undoped GaN film, the spontaneous emission lifetime was prolonged from below 20 to 70 ps by doping with In. Under high-excitation density, stimulated emission was observed from both samples. The threshold excitation density was found to be reduced in the In-doped sample. These significant improvements of the optical properties are attributed to the effective suppression of the formation of the nonradiative recombination centers caused by a change of the growth kinetics induced by a small amount of In supplied during growth of the GaN films. (C) 1999 American Institute of Physics. [S0003-6951(99)00345-9]
\end{abstract}

$\mathrm{GaN}$ and related III-V nitride materials have recently drawn a great deal of attention mainly because of their possible applications in light emitting devices operating in the green, blue, and ultraviolet wavelength regions. InGaN ternary alloys are widely used as the active layer for practical devices such as blue/green light-emitting diodes ${ }^{1}$ and violet laser diodes. ${ }^{2}$ A further extension to shorter wavelength requires the $\mathrm{GaN}$ or $\mathrm{AlGaN}$ as active layers. However, devices realized with these active layers are rare, partly because materials with wide band gaps energy easily suffer from the formation of nonradiative recombination centers which may lead to a deterioration in luminescence efficiency. A solution to this problem may be to use a surfactant. ${ }^{3}$ Surfactants are expected to modify the kinetics of epitaxial growth, therefore choosing the adequate surfactant could result in the improvement of crystal quality by controlling the surface diffusion length of the adatoms. Recently, some experimental reports on In surfactant effects investigated from a viewpoint of optical, ${ }^{4-6}$ electrical, ${ }^{6}$ and growth properties ${ }^{7}$ have been published. All those studies showed positive effects of In, supplied on the surfaces during growth, to improve the crystal quality of GaN.

In this study, we demonstrate the effect of In doping on the improvement of crystal quality by performing timeresolved photoluminescence (TRPL) spectroscopy. Because the carrier lifetime reflects the concentration of recombination centers directly, this investigation method provided us with a sensitive probe for detection of nonradiative recombination centers. The excitation density was chosen in the range where the corresponding photogenerated carrier density was around and above the Mott density. This allowed us to explore the optical properties under a high carrier density similar to what is found in commercial devices operating under high carrier injection.

In-doped GaN films as well as undoped films (both approximately $0.4 \mu \mathrm{m}$ thick) were prepared by gas-source

${ }^{a)}$ Electronic mail: kumano@es.hokudai.ac.jp molecular-beam epitaxy (GS-MBE) on low-temperature grown $\mathrm{GaN}$ buffer $/ \mathrm{Al}_{2} \mathrm{O}_{3}$ substrate. Details about the growth procedure can be found in Ref. 4. Both samples have $n$-type conductivity with an electron concentration of $10^{18}-10^{19} \mathrm{~cm}^{-3}$ at room temperature. ${ }^{6}$ From the absence of energy shift of the band edge-related PL emission, the In incorporation was estimated to be less than $0.1 \%$. $^{4,6}$ To study the steady-state PL properties, PL measurement excited by a $\mathrm{He}-\mathrm{Cd}(325 \mathrm{~nm})$ laser at $14 \mathrm{~K}$ was carried out and spectra were shown in the lower part of Figs. 1(a) and 1(b). The peak at $3.47 \mathrm{eV}$ which is attributed to the neutral donor bound exciton $\left(I_{2}\right)^{8}$ was observed for both samples but the PL emissions related to the impurity or structural defects (3.43 $\mathrm{eV})$ and the donor to acceptor pair emission $(3.32 \mathrm{eV})$ were decreased or absent in the In-doped GaN films. Furthermore, the intensity of the $I_{2}$ emission was enhanced about 60 times by the In doping. These features are attributed to a reduction of the impurity incorporation and/or a reduced formation of defects due to a modification of the growth kinetics induced by an In supply during growth.

To further investigate the effects of In doping, femtosecond TRPL experiments were performed using a SpectraPhysics mode-locked Ti:sapphire laser followed by a regenerative amplifier (REGEN) operating at $1 \mathrm{kHz}$. The REGEN was seeded by a mode-locked $80 \mathrm{fs} / 800 \mathrm{~nm}$ laser pulse. The output of the REGEN had a photon energy of $1.55 \mathrm{eV}$, and was sent to an optical parametric amplifier. An excitation pulse $(3.79 \mathrm{eV})$ with a pulse duration of about 200 fs was obtained after a parametric amplification and a fourth harmonic generation process. The spot diameter of the excitation pulse on the sample surface was estimated to be 200 $\mu \mathrm{m}$. Photon counting with a high-speed single-scan streak camera (Hamamatsu C5680) was used to detect the luminescence. The overall time resolution including the laser pulse width and the temporal spread within the monochromator was $<20 \mathrm{ps}$. All the TRPL spectra were detected at $8 \mathrm{~K}$ vertical to the epilayer surface.

Solid lines in Fig. 2 show the time decay profiles of both 


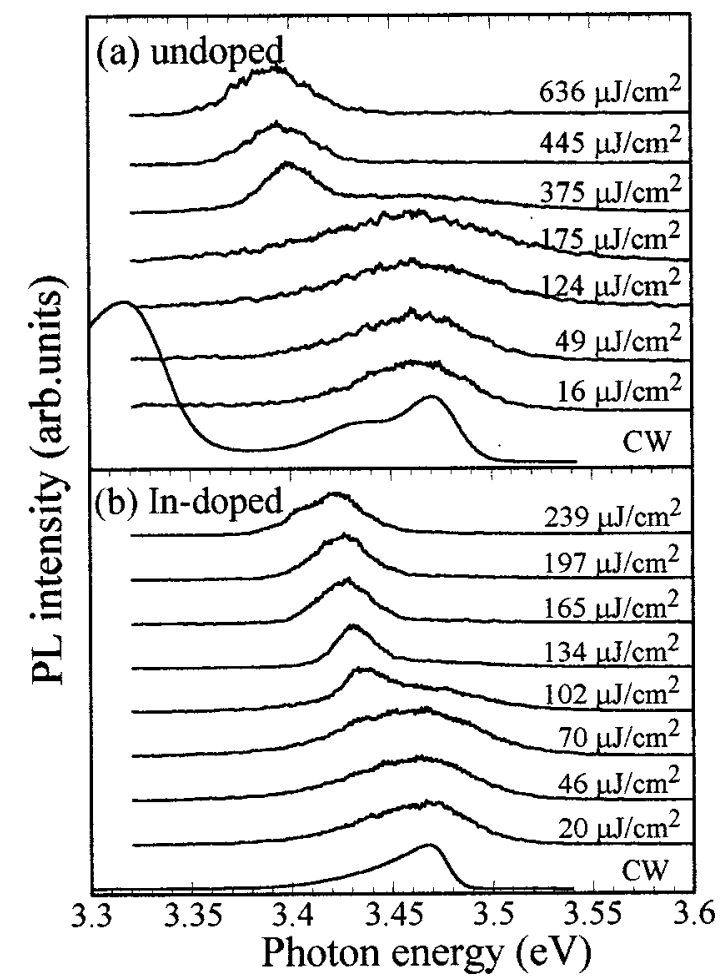

FIG. 1. cw excited PL (lowest spectra) and TRPL spectra at various excitation densities for both undoped (a) and In-doped (b) GaN films. The sample temperature during the $\mathrm{cw}$ and the transient PL measurements were 14 and $8 \mathrm{~K}$, respectively. All TRPL spectra are normalized.

films at the lowest excitation density. In-doped $\mathrm{GaN}$ has longer lifetime of $70 \mathrm{ps}$ whereas the undoped GaN has the lifetime of 20 ps. Considering with the improved film quality observed by continuous wave (cw)-PL, this increased lifetime strongly indicates that indium supply effectively eliminates the formation of the nonradiative recombination centers.

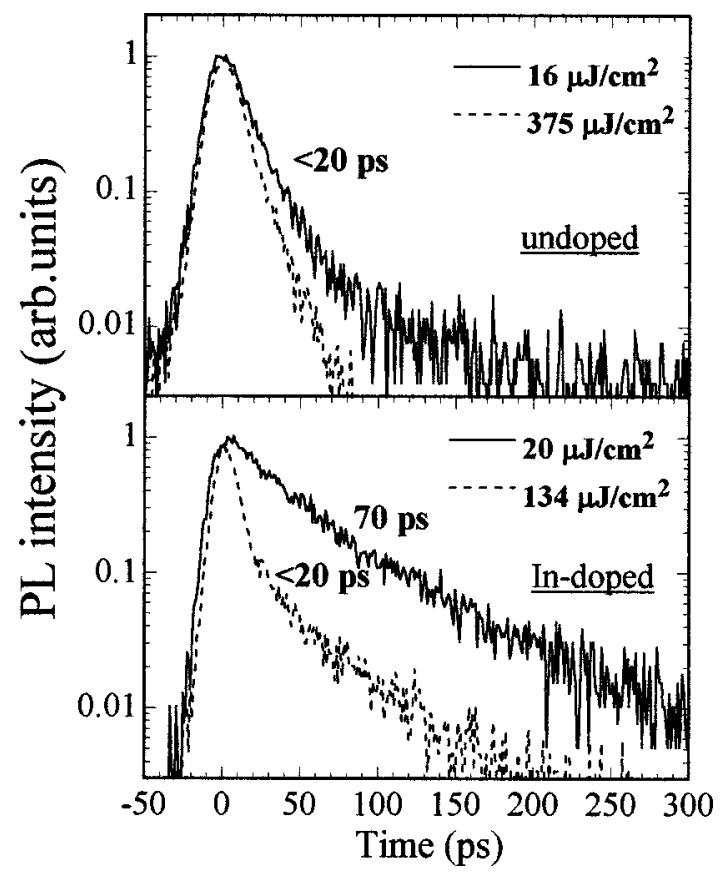

FIG. 2. Decay profiles of the two different excitation densities. At the lowest excitation density $\left(\Phi_{\mathrm{ex}}\right)$ (solid lines), the lifetime is clearly prolonged by In doping. At the higher $\Phi_{\mathrm{ex}}$ above the threshold, rapid decrease of lifetime is observed in In-doped GaN.

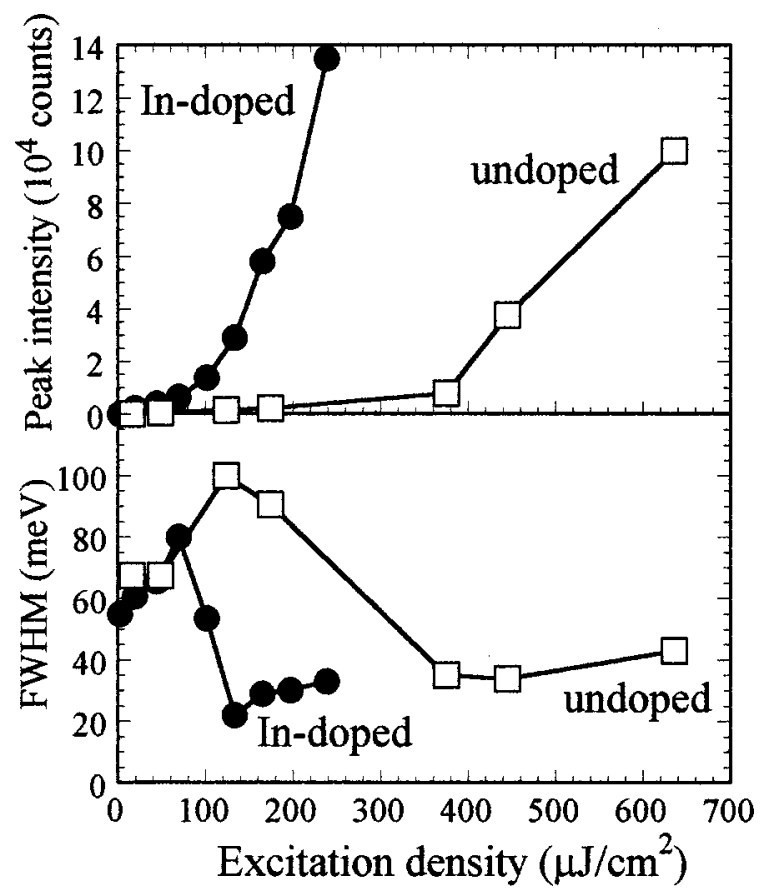

FIG. 3. Excitation density dependence of the peak intensity (upper curves) and the FWHM (lower curves) as obtained from TRPL spectroscopy. The threshold excitation density was found to be approximately $380 \mu \mathrm{J} / \mathrm{cm}^{2}$ for undoped and $140 \mu \mathrm{J} / \mathrm{cm}^{2}$ for In-doped $\mathrm{GaN}$.

Figure 1 shows the excitation density $\left(\Phi_{\mathrm{ex}}\right)$ dependence of time-integrated PL spectra. Using absorption coefficient of $1.2 \times 10^{5} \mathrm{~cm}^{-1}$ (Ref. 9) and a reflection coefficient of $10 \%$ measured at the laser wavelength, photogenerated carrier density is estimated to be $2.8 \times 10^{18} \mathrm{~cm}^{-3}$ at $16 \mu \mathrm{J} / \mathrm{cm}^{2}$ excitation density. With the increase of the excitation density, full width at half maximum (FWHM) of PL spectra were broadened. When the excitation exceeded a certain value, the peak shifted to the lower energy accompanied with narrowing of the FWHM as shown in Fig. 1. The absolute intensity and the FWHM as a function of excitation density are plotted in Fig. 3. A linear increase followed by superlinear increase of PL the peak intensity can be seen. The break point is $380 \mu \mathrm{J} / \mathrm{cm}^{2}$ for the undoped and $140 \mu \mathrm{J} / \mathrm{cm}^{2}$ for In-doped $\mathrm{GaN}$. Above the breakpoint the lifetime of the In-doped GaN film shows decrease from 70 to $<20 \mathrm{ps}$ as shown by the dashed line in Fig. 2. For the undoped GaN, a lifetime shortening could not be observed because the temporal resolution of the detection system limited the measurements. These behaviors of an abrupt increase of the PL intensity, a rapid decrease of the FWHM of the PL peak, and the shortening of the carrier lifetime above a certain excitation density are all characteristic features of the onset of stimulated emission (SE). In our experiment, luminescence was collected from a direction normal to the sample surface. It is likely that the observed stimulated emission originates from the light amplification during the propagation along the sample surface, followed by the scattering due to remaining surface roughness. ${ }^{10-12}$ In-doped GaN has reduced threshold by approximately three times in comparison to undoped $\mathrm{GaN}$. This corresponds reasonably to the longer spontaneous emission lifetime of In-doped $\mathrm{GaN}$ below the threshold since the longer carrier lifetime requires the lower excitation density to achieve the population inversion. 
After the onset of stimulated emission, the peak energy shifts to the lower energy and the FWHM becomes broader as the excitation density is increased. In this excitation region, the concentration of photogenerated carriers completely exceeds the Mott density of $8 \times 10^{17} \mathrm{~cm}^{-3},{ }^{13}$ and therefore, the electron-hole plasma will be responsible for this stimulated emission. This interpretation is also consistent with the observed relatively large energy separation between the spontaneous and stimulated emissions $(63 \mathrm{meV}$ for undoped and $35 \mathrm{meV}$ for In-doped) which cannot be accounted for by the participation of the excitons in the SE mechanism. The origin of the different redshift observed in two samples is possibly attributed to the larger selfabsorption and internal losses, ${ }^{13}$ and the larger band gap renormalization due to higher carrier density in the undoped $\mathrm{GaN}$. Further study is required to clarify this point.

In summary, we have reported a prolonged lifetime of the carriers and a lowering the threshold excitation density for stimulated emission by doping GaN films with In. The measurements were carried out by means of TRPL spectroscopy on GaN grown on sapphire substrates by GS-MBE. The experimental results were explained by an improvement in crystal quality caused by the effective suppression of nonradiative recombination centers due to a modification of growth kinetics induced by a small amount of In supplied during the growth.
This work was supported in part by a Grant-in Aid for Scientific Research from the Ministry of Education, Science and Culture, No. 11750246.

${ }^{1}$ S. Nakamura, M. Senoh, N. Iwasa, S. Nagahama, T. Yamada, and T. Mukai, Jpn. J. Appl. Phys., Part 2 34, L1332 (1995).

${ }^{2}$ S. Nakamura, M. Senoh, S. Nagahama, N. Iwasa, T. Yamada, T. Matsushita, Y. Sugimoto, and H. Kiyoku, Appl. Phys. Lett. 70, 2753 (1997).

${ }^{3}$ M. Copel, M. C. Reuter, E. Kaxiras, and R. M. Tromp, Phys. Rev. Lett. 63, 632 (1989).

${ }^{4}$ X. Q. Shen and Y. Aoyagi, Jpn. J. Appl. Phys., Part 2 38, L14 (1999).

${ }^{5}$ C. K. Shu, J. Ou, H. C. Lin, W. K. Chen, and M. C. Lee, Appl. Phys. Lett. 73, 641 (1998).

${ }^{6}$ X. Q. Shen, P. Ramvall, P. Piblet, and Y. Aoyagi, Jpn. J. Appl. Phys., Part 2 38, L411 (1999).

${ }^{7}$ F. Widmann, B. Daudin, G. Feuillet, N. Pelekanos, and J. L. Rouvière, Appl. Phys. Lett. 73, 2642 (1998).

${ }^{8}$ S. J. Rhee, S. Kim, E. E. Reuter, and S. G. Bishop, Appl. Phys. Lett. 73, 2636 (1998).

${ }^{9}$ J. F. Muth, J. H. Lee, I. K. Shmagin, R. M. Kolbas, H. C. J. Casey, B. P. Keller, U. K. Mishra, and S. P. DenBaars, Appl. Phys. Lett. 71, 2572 (1997).

${ }^{10}$ M. A. Khan, D. T. Olson, J. M. Van Hove, and J. N. Kuznia, Appl. Phys. Lett. 58, 1515 (1991).

${ }^{11}$ D. Wiesmann, I. Brener, L. Pfeiffer, M. A. Khan, and C. J. Sun, Appl. Phys. Lett. 69, 3384 (1996).

${ }^{12}$ S. Bidnyk, T. J. Schmidt, G. H. Park, and J. J. Song, Appl. Phys. Lett. 71, 729 (1997).

${ }^{13}$ S. Hess, R. A. Taylor, J. F. Ryan, B. Beaumont, and P. Gibart, Appl. Phys. Lett. 73, 199 (1998) 